\title{
Mythen und Realitäten
}

\section{Steffen Lau, Josef Sachs ${ }^{\mathrm{b}}$}

${ }^{a}$ Dr. med., Chefarzt, stv. Klinikdirektor, Zentrum für Stationäre Forensische Therapie, Klinik für Forensische Psychiatrie, Psychiatrische Universitätsklinik Zürich

${ }^{b}$ Dr. med., Chefarzt, Bereichsleiter Forensische Psychiatrie, Psychiatrische Dienste Aargau

\begin{abstract}
Über Gesetze, welche die Weitergabe von Informationen aus forensischen Therapien an Aufsichtsbehörden regeln sollen, wird kontrovers diskutiert. Dem Argument, ein Arzt sei auch im Vollzug nur dem Patienten verpflichtet und die Offenbarung von Geheimnissen sei ein therapieschädlicher Vertrauensbruch, wird hier widersprochen. Im Zwangskontext durchgeführte Therapien müssen der Überprüfung durch Rechtsinstanzen zugänglich sein.
\end{abstract}

In der SÄZ wird seit einigen Monaten eine Debatte geführt um Sinn (bzw. Unsinn) und Zweck forensischer Therapien und deren Rahmenbedingungen. Darin wird immer wieder ein Aspekt gestreift, der auch nicht-forensisch tätige Psychiater zu pointierten Positionen bewegt - die Frage des Umgangs mit der ärztlichen Schweigepflicht in der behandlungsorientierten forensischen Psychiatrie. Dass es möglicherweise gute Gründe dafür gibt, das in anderen medizinisch-therapeutischen Zusammenhängen sinnvoll eingesetzte Prinzip der Verschwiegenheit des Arztes aufzugeben, scheint den Berufsstand nicht kalt zu lassen. Als Bei-

Le secret professionnel en psychiatrie forensique - mythes et réalités

La nécessité d'édicter des lois devant régler la question de la transmission d'informations issues de thérapies forensiques aux autorités de surveillance fait l'objet de débats controversés. D'une part, certains avancent que le traitement de délinquants souffrant de maladie psychique n'a pas lieu dans le cadre d'une relation contractuelle de droit privé, et qu'il existe donc un intérêt légitime justifiant la transmission d'informations. De l'autre, on estime qu'un médecin n'a de devoirs qu'envers son patient, y compris en détention, et qu'une divulgation de secrets constitue une rupture de confiance qui compromet l'efficacité de la thérapie. Le présent article s'emploie à réfuter ce dernier argument. II convient en effet de distinguer entre une thérapie ordonnée par un tribunal et une thérapie à laquelle se soumet spontanément une personne détenue. Dans ces cas-là aussi, il existe un devoir d'information si la thérapie est axée sur la prévention du délit. Toutefois, lors de mesures thérapeutiques à proprement parler, le devoir de rendre régulièrement compte fait partie intégrante de la thérapie, et doit être communiqué d'emblée au patient en toute transparence. La transparence ne compromet en aucun cas l'efficacité du traitement. Par ailleurs, les thérapies réalisées dans un contexte de contrainte doivent pouvoir être examinées par les instances juridiques. Or cela n'est possible que si l'on a le droit de divulguer ce qui s'est passé ou doit se passer dans l'espace protégé de la thérapie. spiel dafür sei an das Editorial der Schweizerischen Ärztezeitung vom 3. September 2014 erinnert. Die Präsidentin des Walliser Ärzteverbandes forderte darin, dass unter dem Eindruck der Morde an Adeline und Marie in den Kantonen Waadt und Genf unter politischem Druck keine "emotionsbasierten» Gesetzesänderungen umgesetzt werden sollten [1]. Insbesondere wurde eine als sinnvoll erachtet Meldepflicht kritisiert für Ärzte, die in die Behandlung von als gefährlich eingestuften Straftätern involviert sind. Dass eine $\mathrm{Ge}$ sundheitsfachperson «die zuständigen Verwaltungsund Justizbehörden über alle wichtigen Tatsachen zu informieren hat, welche einen Einfluss auf die laufenden Massnahmen [...] oder im Allgemeinen auf die Beurteilung der Gefährlichkeit der betroffenen Personen haben können», wurde als unverhältnismässig und kontraproduktiv erachtet. Und später formulierte der Kollege Mario Gmür [2] (allerdings nicht unwidersprochen [3]) die Forderung nach uneingeschränkter Gewährleistung des Arztgeheimnisses zugunsten von Massnahmepatienten. Diese Haltung ist nicht neu, schon seit langer Zeit [4] wird argumentiert, dass ein Arzt auch im Massnahmenvollzug nur dem Patienten verpflichtet sei und eine Offenbarung von Geheimnissen ohne Einverständnis des Patienten einen schweren Vertrauensbruch darstelle, eine Generalvollmacht genüge nicht, erforderlich für eine Informationsweitergabe betreffend die Therapie sei eine neue und ausdrückliche Einwilligung des Patienten.

Auch für eine Gegenposition finden sich gute Argumente: Die Behandlung psychisch kranker Rechtsbrecher findet nicht innerhalb eines privatrechtlich geregelten Vertragsverhältnisses statt, sondern in einem öffentlichen Kontext. Patient, Therapeut, Justiz, politische Entscheidungsträger und Öffentlichkeit bilden das System der Behandlung, was etwas gänzlich ande- 
res ist als die vertraute Arzt-Patienten-Dyade. Bei der Behandlung von Massnahmenpatienten ist sowohl der Patient als auch die Vollzugsbehörde Auftraggeber, somit steht der Arzt bei beiden in der Verantwortung. Wenn man denn in diesem Zusammenhang "emotionsbasierte» (s.o.) Gesetzesänderungen kritisiert, so sollte man sich jedoch auch nicht unrealistischen Vorstellungen über die Behandlung von gefährlichen Straftätern hingeben. Diese wirken auf den forensisch erfahrenen Behandler wie romantisierende Ansichten zum tatsächlichen Verhältnis zwischen Straftäter und forensisch tätigem Therapeuten. Offenbar besteht die Vorstellung, dass bei einer hochproblematischen Gruppe von Patienten (die nämlich schon einmal eine gerichtlich festgestellte erhebliche Gefährlichkeit entwickelt hatten) allein die Wahrung des Arztgeheimnisses den Aufbau einer wirksamen therapeutischen Beziehung ermöglicht und dass dies allein die Grundlage schaffe für eine Bearbeitung deliktrelevanter Probleme. Die Realität der Arbeit in der behandlungsorientierten forensischen Psychiatrie sieht allerdings völlig anders aus. Wer Erfahrung hat mit der Behandlung dieser oben beschriebenen gefährlichen Menschen, der erlebt sehr selten, dass von Straftätern Dinge verschwiegen werden, allein weil der Behandler zu einer Offenbarung gegenüber den Justizbehörden verpflichtet wäre. Bei Straftätern, bei denen ein Mangel an Offenheit besteht (sei es nun als Ausdruck gravierender Persönlichkeitspathologie oder als Symptom einer psychotischen Erkrankung), ist diese Problematik meist seit langem bekannt und wird nicht erst im Zeitpunkt der Therapieaufnahme manifest. Sie ist häufig Ausdruck einer zugrundeliegenden dissozialen Problematik und tritt nicht erst durch institutionelle Rahmenbedingungen zu Tage. Es ist gerade eine besondere Kunst, im Rahmen forensischer Therapien mit dem Zugewiesenen darüber zu reflektieren, dass die involvierten Ärzte neben der primären Aufgabe als Therapeut auch noch als Administrator tätig werden und eben auch als Berichterstatter fungieren könnten, und dies auch offensiv zu vertreten [5]. Entscheidend ist allerdings, dass der Umgang mit der Schweigepflicht auch dem Patienten transparent dargelegt wird. Er muss bereits zu Beginn der Therapie wissen, worauf er sich einlässt, und welche Informationen aus der Therapie wem weitergeleitet werden. Diese Transparenz ist nicht nur therapeutisch hilfreich, sondern durchaus auch vertrauensfördernd. Ein solches Vorgehen bedeutet keineswegs zwangsläufig eine Verhinderung von relevanten risikosenkenden Veränderungsprozessen.

Viel wichtiger jedoch: Welchen Sinn hat denn ein Pochen auf die Wahrung eines wie auch immer gearte- ten "Geheimnisses» eines Straftäters? Er selber hat sich doch zuvor schon durch die Begehung einer Straftat vollständig "offenbart». In einer Gerichtsverhandlung wird dann das zu beurteilende Geschehen erneut schonungslos betrachtet. Im Rahmen eines kompetent durchgeführten Strafverfahrens ist die Ausleuchtung der Tat und des inneren Erlebens eines Straftäters unter anderem durch eine forensisch-psychiatrische Begutachtung und Gefährlichkeitseinschätzung unverzichtbar. Das muss in einem Täterstrafrecht auch so sein, um einem Angeklagten in einem Strafprozess «gerecht» zu werden. Nur bei genauer Kenntnis des Bedingungsgefüges der Straffälligkeit eines Menschen kann die gerichtliche Beurteilung eine «verhältnismässige» Reaktion des Staates (u.a. die Anordnung einer sog. Behandlungsmassnahme) zur Folge haben.

\section{Der Umgang mit der Schweigepflicht muss dem Patienten transparent dargelegt werden.}

Folgt man den Anhängern einer absoluten Verschwiegenheitspflicht im Straf- und Massnahmenvollzug, so soll ab dem Beginn einer deliktpräventiven Therapie in einer Vollzugseinrichtung keine offene Kommunikation mehr zwischen den von Gesetzes wegen Beteiligten über Veränderungen im Bedingungsgefüge der Delinquenz stattfinden, weil diese Kommunikation nun nicht mehr konstruktiv, sondern sogar schädlich sei. Kaum ist ein Straftäter rechtskräftig verurteilt, soll demnach der Mantel des Schweigens über den Prozess der Beeinflussung des von ihm ausgehenden Risikos gebreitet werden. Wie soll unter solchen Voraussetzungen dann die berechtigte Frage der Vollzugsbehörden, ob die Faktoren der Gefährlichkeit eines Straftäters ausreichend beeinflusst wurden, beantwortet werden? Soll man als forensischer Therapeut gegenüber den Behörden (dem eigentlichen Auftraggeber) lediglich ein pauschales Urteil formulieren wie «Er ist soweit» oder aber "Er braucht noch Zeit»? Wie soll die Institution, die den Behandlungsprozess auch überwachen soll, überprüfen, dass eine Einschätzung des Behandlungsverlaufs aus der Behandlungsinstitution nicht lediglich intuitiv getroffen wurde, sondern auf einer nachvollziehbaren Veränderung der straftatfördernden Problembereiche beruht?

Grundsätzlich muss im Straf- und Massnahmenvollzug unterschieden werden zwischen der im Rahmen einer therapeutischen Massnahme gerichtlich angeordneten deliktpräventiven Therapie einerseits und einer Therapie, der sich eine Person im Strafvollzug freiwillig unterzieht. Bei der Massnahmentherapie ist die Pflicht zur regelmässigen Berichterstattung unverzichtbarer Bestandteil der Therapie, denn die Ausgestaltung des Voll- 
zugs hängt unter anderem vom Therapieverlauf $a b$. Dem gegenüber werden freiwillige vollzugsbegleitende Therapien nicht angeordnet, sondern auf Initiative des gefangenen Patienten aufgenommen. Auch in diesen Fällen besteht aber gemäss bundesgerichtlicher Rechtsprechung in einem bestimmten Rahmen eine Informationspflicht, auf die im Behandlungsvertrag eingegangen werden muss [6].

\section{Man sollte sich nicht unrealistischen Vorstel- lungen über die Behandlung von gefährlichen Straftätern hingeben.}

Immer wieder wird forensischen Psychiatern (teilweise zu Recht) vorgeworfen, als "Halbgötter in Weiss" zu agieren, weil sie nicht ausreichend transparent machten, wie sie zu einer Einschätzung gelangen. Durch Forderung eines letztlich eine gefährliche Intransparenz fördernden Vorgehens wird dieser Eindruck wieder verstärkt, obwohl sich in den letzten Jahren eine positive Entwicklung abzeichnete. Die forensische Psychiatrie hat sich nach dem grossen Vertrauensverlust, der u.a. bedingt war durch spektakuläre Straftaten, begangen durch massnahmeerfahrene Rückfalltäter, in den letzten Jahren um Qualitätssteigerung und Qualitätssicherung von Begutachtung und Therapie bei Straftätern bemüht [7]. Ein wesentlicher Meilenstein war dabei die Umsetzung der Erkenntnis der empirischen Rückfallforschung, dass gerade der transparente strukturierte Austausch zwischen den an der Massnahmebehandlung beteiligten Institutionen ein wesentliches Merkmal effizienter rückfallpräventiver Therapien darstellt. Gerade weil man in der forensischen Psychiatrie gefährlichen Menschen behandelt, verbietet es sich, hinter verschlossenen Türen zu arbeiten.

Um es noch einmal klarzustellen - es geht bei den vorgeschlagenen Gesetzesänderungen nicht um die Verpflichtung jedes behandelnden Arztes, sich Gedanken darüber zu machen, inwieweit irgendwelche medizinische Einschätzungen oder Feststellungen Gefährlichkeitspotential beinhalten oder nicht. Ärztinnen und Ärzte sollen Informationen weiterleiten über Patienten, die sich im Straf- oder Massnahmenvollzug befinden. Die an den Behandlungen von Straftätern beteiligten Ärzte sollen von einer Bürde entlastet werden. Sie sollen beim Bericht an die Behörden befreit werden von der Überlegung, ob sie bei einem Austausch von Informationen, die risikorelevant sein können, einen Geheimnisbruch begehen oder nicht. Ein nicht kleiner Teil der in der forensischen Psychiatrie tätigen Ärzte erlebt solche Regelungen als sinnvoll. Geklärt wird dadurch eine in einigen Kantonen bestehende uneindeutige Situation. Dies stellt eine Erleichterung einer ver- antwortungsvollen Tätigkeit dar. Nur wenn offensiv und transparent mit den anderen beteiligten Institutionen (keineswegs der Öffentlichkeit!) über den Verlauf einer Kriminaltherapie kommuniziert werden darf, kann auch Verantwortung (bspw. bei bedeutenden Lockerungsschritten oder Entlassungsentscheidungen) adäquat geteilt werden. Dies stellt einen entscheidenden Punkt bei der Wahrung der öffentlichen Sicherheit dar; auch dies ist ein Auftrag, den die forensische Psychiatrie zu erfüllen hat.

Wenn argumentiert wird, dass die "Aushöhlung» des Berufsgeheimnisses einen Menschen in seinen Grundrechten verletze, so wird verkannt, dass ein Rückzug auf die ärztliche Schweigepflicht im Rahmen von Massnahmetherapien ebenfalls ein Grundrecht der Eingewiesenen tangieren kann. Nur wenn gegenüber Aufsichtsbehörden ohne Tabus erklärt werden darf, warum etwas mit einem forensischen Patienten gemacht werden sollte und wie ich beabsichtige, mögliche Veränderung eines Risikos für Straftaten sichtbar zu machen, kann ich in eine konstruktive Auseinandersetzung darüber eintreten, ob ein solches Vorgehen gerechtfertigt ist oder nicht (Stichwort: Verhältnismässigkeit) [8]. Im Zwangskontext durchgeführte Therapien müssen einer Überprüfung durch Rechtsinstanzen zugänglich sein [9]. Das kann nur geschehen, wenn die Behandler zu jeder Zeit darlegen dürfen, was im prinzipiell geschützten Raum der Therapie geschehen ist oder geschehen soll. Die kritisierten Änderungsvorschläge zu den Regelungen der Schweigepflicht stehen somit keineswegs im Widerspruch zu den Bedürfnissen von Straftätern und ihren Behandlern. Schon gar nicht, wenn mit Ihnen die Wahrung eines wesentlichen Prinzips der Rechtsstaatlichkeit verknüpft ist.

\section{Literatur}

1 Lehky Hagen M. Aufweichung des Arztgeheimnisses gefährdet öffentliche Sicherheit. Schweiz Ärztezeitung 2014;95(36):1311.

2 Gmür M. Ethische Wegweiser für Prognosestellung und Psychotherapie im Strafrecht sowie Straf- und Massnahmenvollzug. Schweiz Ärztezeitung 2014;95(36):1341-3.

3 Graf M, Habermeyer E. Die Forensische Psychiatrie ist der Medizin und dem Recht verpflichtet. Schweiz Ärztezeitung 2015;96(12):447-

4 Der Röstigraben beim Arztgeheimnis. NZZ, 3.11.2014. http://www. nzz.ch/schweiz/der-roestigraben-beim-arztgeheimnis-1.18416943

5 Müller-Isberner R, Eucker S. Therapie im Massregelvollzug, Berlin: MWV 2009; S. 99

6 BGE 6B_4/2011.

7 Sachs J, Habermeyer E. Qualifizierung und Qualitätskontrolle in der forensischen Psychiatrie - Die gegenwärtigen Standards in der Schweiz. Forens Psychiatr Psychol Kriminol 2012;6:258-65.

8 Lau S. Therapie der Persönlichkeitsstörungen im Massregelvollzug. In: Lau S, Lammel M, Sutarski S (Hrsg.) Forensische Begutachtung bei Persönlichkeitsstörungen. 2. Aufl., Berlin: MWV, 2014 S. 179.

9 Brägger B. Massnahmenvollzug an psychisch kranken Straftätern in der Schweiz: Eine kritische Auslegeordnung. Schweizerische Zeitschrift für Kriminologie 2014;12(2):36-44. 